\title{
Effect of nitrogen rates and seedling age on the productivity of some promising hybrid rice combinations
}

\author{
Hassan Shehata Hamad \\ Rice Research \& Department, Field Crops Research Institute, Agriculture Research Center, Egypt \\ Corresponding author: Hassanshehata28@yahoo.com
}

\begin{abstract}
This experiment was conducted at Experimental Farm of Rice Research Department at Sakha Agriculture Research Station, Sakha, Kafr EL Sheikh, Egypt during 2014 and 2015 rice growing seasons to study the effect of seedling ages and nitrogen rates on the productivity of two Egyptian hybrids combinations, Egyptian hybrid 1 and new hybrid combination SK2151 H on different characters. A split split-plot design with three replications was used. The main plot was devoted to hybrid rice combinations, while the seedling age were arranged in the sub plots and nitrogen rates were assigned in the sub-sub plots with three replications. Four rates of nitrogen (zero, 55, 110 and $165 \mathrm{~kg} \mathrm{~N} / \mathrm{ha}$ ) and three seedling ages (20, 25 and 30 days), were used, The date of sowing was at $1^{\text {st }}$ May of each season. The data were recorded on plant height $(\mathrm{cm})$, panicles hill ${ }^{-1}$, panicle weight $(\mathrm{g})$, panicle length $(\mathrm{cm})$, grain yield $\left(\mathrm{tha}^{-1}\right)$, 1000-grain weight $(\mathrm{g})$, harvest index $(\%)$ and spikelet fertility $\%$. Results showed that, the high rate of nitrogen significantly influenced on plant height $(\mathrm{cm})$, panicles hill-1 ${ }^{-1}$, panicle weight $(\mathrm{g})$, panicle length $(\mathrm{cm})$, grain yield $\left(\mathrm{tha}^{-1}\right)$, 1000-grain weight $(\mathrm{g})$, harvest index $(\%)$ and spikelet fertility $(\%)$, The highest grain yield (13.600 tha $\left.{ }^{-1}\right)$ resulted from 20 days seedling age and $165 \mathrm{~kg}$ nitrogen per hectare for the Egyptian hybrid 1 in the second season. While, the lowest grain yield (4.230 tha $\left.{ }^{-1}\right)$ was recorded without application of nitrogen fertilizer (control) with seedling age of 30 days for SK $2151 \mathrm{H}$ in the first season.
\end{abstract}

Key words: Hybrid combination, seedling ages, nitrogen rates and grain yield.

\section{Introduction}

In Egypt the average national yield level of rice has to be increased by $25-30 \%$ to meet the demands of the increasing population. This seems difficult considering the narrow gap between yield potential and actual yield (10.5 tha $\left.{ }^{-1}\right)$ in 2013. Among available technologies to increase yield above the present ceiling, is the exploitation of heterosis in hybrid rice, which appears to be the practical approach for Egypt (Bastawisi et al. 1998). The seedling age of rice plants is conventionally expressed in calendar days after germination or seedling. However, the calendar days are much affected by the environment. Several experiments were conducted to study the optimum rice seedling age and concluded that, 25-30 days is the optimum seedling age under all sowing dates regardless of variety and locations (Rice Research Training Center Sakha, Kafr El-Sheikh, Egypt, 2002). Seedling age plays an important role in enhancing the yield of rice. Age of seedling is a key factor which influences the tillers production, grain formation and other yield contributing parameters. It is the main factor for uniform stand establishment of rice which controls its growth and yield. The use of appropriate aged seedlings for transplanting and its timely planting are important non-cash inputs for attaining the higher yield of rice. Seedling vigor contributes to successive tillering, yield and quality of transplanted rice. Growth and production of rice depend on timely cultivation and growth duration of cultivar which is affected by age of seedlings at transplanting. All the above and below ground morphological characters of rice plants were varied with seeding rate, age of seedling and growing environment. Nitrogen plays a key role in rice production as it is required in huge amount. (Waghmode et al, 2008), found that, the mean devotions of delayed $50 \%$ flowering from the recommended age of transplanting ( 25 days old ) was highest for transplanting 40-day old seedlings (by 7.50,7.12and 7.63 days for $\mathrm{A}, \mathrm{B}$, and $\mathrm{R}$ lines, respectively ) flowed by 3 days - old seedling (4.88,4.75 and 5.00 days), 30-days - old seedling (2.25, 2,00 and 2.25 days) and 20 days - old seedlings $(2.25,2.00$ and 2.50 days ).The $50 \%$ flowering was delayed in seedling that were younger or older than the seedling with optimum age $(25$ days old).The complete synchronization of flowering in IR 58025A and B was obtained by 3 staggered sowing of plants during transplanting, the synchronization in $\mathrm{A}$ and $\mathrm{R}$ line was achieved by 3 staggered sowing of $\mathrm{R}$ line at 5days intervals on the $1^{\text {st }}, 6^{\text {th }}$ and $11^{\text {th }}$ day of the month, and of A line on the 23 days of the month due to the effect of overage seedlings of $\mathrm{R}$ line at transplanting. So; this investigation was conducted to study the effect of nitrogen fertilizer rates and seedling age on the productivity of some promising rice hybrids.

\section{Materials and Methods}

This experiment was conducted at Experimental Farm of Rice Research Department at Sakha Agriculture Research Station, Sakha, Kafr EL Sheikh, Egypt, during 2014 and 2015 rice growing seasons 
to study the effect of nitrogen rates ( zero, 55, 110 and $165 \mathrm{~kg} \mathrm{~N} / \mathrm{ha}$ ) and three seedling age (20, 25 and 30 days ) for two hybrid rice combination, Egyptian hybrid 1 and new hybrid combination SK2151 H on plant height $(\mathrm{cm})$, panicles hill ${ }^{-1}$, panicle weight $(\mathrm{g})$, panicle length $(\mathrm{cm})$, grain yield $\left(\right.$ tha $\left.^{-1}\right)$, 1000-grain weight $(\mathrm{g})$, harvest index $(\%)$ and spikelet fertility $(\%)$. The date of sowing was on $1^{\text {st }}$ May of each season. Seedlings were transplanted in plots $3 \times 5 \mathrm{~m}$ in dimensions at $20 \times 20 \mathrm{~cm}$ planting spacing among hills and rows at the rate of 3 seedlings / hill, Nitrogen fertilizer as urea form $46 \% \mathrm{~N}$ was applied at 55,110 and $165 \mathrm{~kg} / \mathrm{ha}$ as follows:1/3 basal in the dry soil during land preparation before flooding, 1/3 at panicle initiation ( 30 days after transplanting ) and the last dose (1/3) was applied seven days before heading (66 days after transplanting ). A split- split plot design with three replications was used. The main plot was devoted two hybrids. While the seedling ages were arranged in the sub - plot and nitrogen rates was assigned in the sub - sub plots. Observation were recorded on ten plants plots ${ }^{-1}$ taken at random from each hybrid rice combinations in each replication for plant height $(\mathrm{cm})$, panicles hill ${ }^{-1}$, panicle weight $(\mathrm{g})$, panicle length $(\mathrm{cm})$, grain yield $\left(\right.$ tha $\left.^{-1}\right), 1000$-grain weight $(\mathrm{g})$, harvest index $(\%)$ and spikelet fertility (\%). The data were collected according to Standard Evaluation System of IRRI 2014, for all the studied characters, all cultural practices were followed as recommended. The data were analyzed following the ANOVA technique and the mean differences were adjusted by the Duncan's Multiple Range Test, Gomez and Gomez, 1984, using a statistical computer package MSTAT.

\section{Results and Discussion}

The results in Table (1) revealed that, the effect of hybrid rice combinations, seedling age and nitrogen fertilizer, as well as, their interaction on plant height $(\mathrm{cm})$, panicles hill ${ }^{-1}$, panicle weight $(\mathrm{g})$, panicle length $(\mathrm{cm})$, grain yield $\left(\right.$ tha $\left.^{-1}\right)$, 1000-grain weight $(\mathrm{g})$, harvest index $(\%)$ and spikelet fertility $(\%)$. The results showed that, all traits were highly significant affected by hybrid combinations during the two seasons. The highest values were plant height $(\mathrm{cm})$, panicles hill $^{-1}$, panicle weight $(\mathrm{g})$, panicle length $(\mathrm{cm})$, grain yield $\left(\mathrm{tha}^{-1}\right)$, harvest index $(\%)$ and spikelet fertility $(\%)$ were102.30 (cm), 21.20 hill $^{-1}$, $4.29(\mathrm{~g}), 22.68(\mathrm{~cm}), 10.36\left(\mathrm{tha}^{-1}\right), 48.13(\%)$ and $96.52(\%)$ for the seven traits in the second season, respectively. With Egyptian hybrid 1. While the lowest values for these traits obtained when used the hybrid Sk2151H during 2014 season. The highest value of 1000-grain weight was 26.53 (g) with using the hybrid SK2151H during season of 2015, while the lowest value was 25.23 (g) obtained from using the Egyptian hybrid 1 during 2014 season. This revealed the effect of seedling ages influence on plant height $(\mathrm{cm})$, panicles hill-1 ${ }^{-1}$ panicle weight $(\mathrm{g})$, panicle length $(\mathrm{cm})$, grain yield $\left(\right.$ tha $\left.^{-1}\right)$, 1000-grain weight $(\mathrm{g})$, harvest index $(\%)$ and spikelet fertility $(\%)$. Were highly significant affected by seedling age during the both seasons, the highest values were $100.59(\mathrm{~cm}), 22.54\left(\right.$ hill $\left.^{-1}\right), 4.48(\mathrm{~g}), 23.06(\mathrm{~cm})$, $10.656\left(\right.$ tha $\left.^{-1}\right), 27.23(\mathrm{~g}), 50.20(\%)$ and $97.00(\%)$ for mention traits, respectively with using 20 days of seedling age during 2015 season. While the lowest values obtained when using the seedling age 30 days during 2014 season.

Faghani et al, ( 2011) found the significant effect of seedling age on tillering pattern, maximum tillers hill-1 (16.3) were recorded by transplanting 25 days old seedlings while 35 days old seedlings gave minimum tillers hill-1 (15.3) on the other side, Molla (2002) . Found that, twenty-eight days old seedling produced more tillers, panicles/ $\mathrm{m}^{2}$ and grain yield than 21- day's old seedling.

Also, there were significant differences among the nitrogen rates for the studied characters, plant height $(\mathrm{cm})$, number of panicles hill ${ }^{-1}$, panicle weight $(\mathrm{g})$, panicle length $(\mathrm{cm})$, grain yield $\left(\right.$ tha $\left.^{-1}\right)$, 1000grain weight $(\mathrm{g})$, harvest index $(\%)$ and spikelet fertility (\%). in both seasons. The highest values were $108.77(\mathrm{~cm}), 26.83$ hill $^{-1}, 4.80(\mathrm{gm}), 24.50$ $(\mathrm{cm}), 11.780\left(\right.$ tha $\left.^{-1}\right), 27.94(\mathrm{~g}), 53.26(\%)$ and 98.02 (\%) for all traits in the second season, respectively with using the rates of $165 \mathrm{~kg} \mathrm{~N} / \mathrm{ha}$. But the lowest values obtained for zero nitrogen rates for all traits during 2014 season.

Also, the result shown in Table (1) indicates that, all interactions among the three factors were highly significant for all the studied traits during the two seasons.

The results in (Table 2) indicated that, the Egyptian Hybrid 1 with the seedling age of 20 days gave significantly higher values for plant height $(\mathrm{cm})$, number of panicles hill ${ }^{-1}$, panicle weight $(\mathrm{gm})$, panicle length $(\mathrm{cm})$, grain yield $\left(\right.$ tha $\left.^{-1}\right)$, 1000-grain weight $(\mathrm{gm})$, harvest index $(\%)$ and spikelet fertility (\%) were $104.10(\mathrm{~cm}), 23.11$ hill $^{-1}, 4.70(\mathrm{~g})$, 23.53(cm), $11.343\left(\right.$ tha $\left.^{-1}\right), 51.22(\%)$ and $96.28(\%)$ for these traits in the second season, respectively, with Egyptian Hybrid 1 under recorded old seedling 20 days. While, significantly lowest values were detected as a result of 30 days old seedling under with hybrid SK2151H for these traits in both seasons. The highest value of 1000-grain weight was 26.75 (g) when using seedling age of 20 days with the hybrid rice combination SK2151H during season 2015 , but, the lowest value was 22.95 (g) obtained by of 30 days seedling age with Egyptian Hybrid 1 during 2015 season. 
Table 1. Effect of hybrid combination, seedling age and nitrogen rates, as well as, their interaction on plant height (cm), panicles hill-1 ${ }^{-1}$ panicle weight ( $\mathrm{g}$ ), panicle length $(\mathrm{cm})$, grain yield $\left(\mathrm{tha}^{-1}\right), 1000$-grain weight $(\mathrm{g})$, harvest index $(\%)$ and spikelet fertility (\%).

\begin{tabular}{|c|c|c|c|c|c|c|c|c|c|c|c|c|c|c|c|c|}
\hline \multirow{2}{*}{$\begin{array}{l}\text { Main effect and } \\
\text { interaction }\end{array}$} & \multicolumn{2}{|c|}{ Plant height $(\mathrm{cm})$} & \multicolumn{2}{|c|}{ Panicals hill ${ }^{-1}$} & \multicolumn{2}{|c|}{$\begin{array}{l}\text { Panicle weight } \\
\text { (g) }\end{array}$} & \multicolumn{2}{|c|}{$\begin{array}{l}\text { Panicle length } \\
(\mathrm{cm})\end{array}$} & \multicolumn{2}{|c|}{ Grain yield $\left(\right.$ tha $\left.^{-1}\right)$} & \multicolumn{2}{|c|}{$\begin{array}{l}\text { 1000-grain } \\
\text { weight }(\mathrm{g})\end{array}$} & \multicolumn{2}{|c|}{$\begin{array}{c}\text { Harvest Index } \\
(\%)\end{array}$} & \multicolumn{2}{|c|}{$\begin{array}{l}\text { Spikelet fertility } \\
(\%)\end{array}$} \\
\hline & 2014 & 2015 & 2014 & 2015 & 2014 & 2015 & 2014 & 2015 & 2014 & 2015 & 2014 & 2015 & 2014 & 2015 & 2014 & 2015 \\
\hline \multicolumn{17}{|l|}{ Hybrids } \\
\hline Egyptian Hybrid 1 & $100.03 a$ & $102.30 \mathrm{a}$ & $19.85 \mathrm{a}$ & $21.20 \mathrm{a}$ & $4.002 \mathrm{a}$ & $4.29 \mathrm{a}$ & $21.82 \mathrm{a}$ & $22.68 \mathrm{a}$ & $9.540 \mathrm{a}$ & $10.361 \mathrm{a}$ & $25.23 b$ & $25.73 b$ & $41.81 \mathrm{a}$ & $48.13 \mathrm{a}$ & $94.40 \mathrm{a}$ & $96.52 \mathrm{a}$ \\
\hline SK2151 H & $94.08 \mathrm{~b}$ & $96.16 \mathrm{~b}$ & $18.68 \mathrm{~b}$ & $20.08 b$ & $3.57 \mathrm{~b}$ & $3.87 \mathrm{~b}$ & $21.21 \mathrm{~b}$ & $21.74 b$ & $8.580 \mathrm{~b}$ & $9.221 \mathrm{~b}$ & $25.91 \mathrm{a}$ & $26.53 a$ & $40.86 b$ & $46.29 b$ & $93.65 b$ & $95.66 \mathrm{~b}$ \\
\hline F-test & $* *$ & $* *$ & $* *$ & $* *$ & $* *$ & $* *$ & $* *$ & $* *$ & $* *$ & $* *$ & $* *$ & $* *$ & $* *$ & $* *$ & $* *$ & $* *$ \\
\hline \multicolumn{17}{|l|}{ Seedling ages } \\
\hline 20 days & $98.45 \mathrm{a}$ & $100.59 a$ & $20.99 a$ & $22.54 \mathrm{a}$ & $4.11 \mathrm{a}$ & $4.48 \mathrm{a}$ & $22.10 \mathrm{a}$ & $23.06 \mathrm{a}$ & $10.027 \mathrm{a}$ & $10.656 a$ & $26.09 \mathrm{a}$ & $27.23 \mathrm{a}$ & $43.02 \mathrm{a}$ & $50.20 \mathrm{a}$ & $95.01 \mathrm{a}$ & $97.00 \mathrm{a}$ \\
\hline 25 days & $96.97 b$ & $99.45 \mathrm{a}$ & $19.36 \mathrm{~b}$ & $20.64 b$ & $3.80 \mathrm{~b}$ & $4.05 \mathrm{~b}$ & $21.56 \mathrm{~b}$ & $22.25 \mathrm{~b}$ & $9.079 \mathrm{~b}$ & $9.808 \mathrm{~b}$ & $25.68 b$ & $25.67 \mathrm{~b}$ & $41.33 b$ & $46.92 b$ & $94.29 b$ & $96.30 \mathrm{~b}$ \\
\hline 30 days & $95.74 \mathrm{c}$ & $97.64 b$ & $17.45 \mathrm{c}$ & $18.75 \mathrm{c}$ & $3.44 \mathrm{c}$ & $3.71 \mathrm{c}$ & $20.88 \mathrm{c}$ & $21.33 \mathrm{c}$ & $8.087 \mathrm{c}$ & $8.908 \mathrm{c}$ & $24.93 \mathrm{c}$ & $25.00 \mathrm{c}$ & $39.65 \mathrm{c}$ & $44.51 \mathrm{c}$ & $92.78 \mathrm{c}$ & $94.96 \mathrm{c}$ \\
\hline F-test & $* *$ & $* *$ & $* *$ & $* *$ & $* *$ & $* *$ & $* *$ & $* *$ & $* *$ & $* *$ & ** & $* *$ & ** & ** & $* *$ & ** \\
\hline \multicolumn{17}{|l|}{ N-rates (kg N/ha) } \\
\hline 0 & $89.31 d$ & $91.10 d$ & $11.67 d$ & $13.23 \mathrm{~d}$ & $3.27 \mathrm{~d}$ & $3.46 \mathrm{~d}$ & $17.77 \mathrm{~d}$ & $19.11 d$ & $5.00 \mathrm{~d}$ & $5.800 \mathrm{~d}$ & $24.27 \mathrm{~d}$ & $24.80 \mathrm{~d}$ & $32.12 d$ & $32.56 \mathrm{~d}$ & $91.95 \mathrm{~d}$ & $94.02 d$ \\
\hline 55 & $94.36 \mathrm{c}$ & $96.09 \mathrm{c}$ & $17.84 \mathrm{c}$ & $18.74 \mathrm{c}$ & $3.48 \mathrm{c}$ & $3.65 \mathrm{c}$ & $21.76 \mathrm{c}$ & $22.14 \mathrm{c}$ & $9.730 \mathrm{c}$ & $10.510 \mathrm{c}$ & $25.13 \mathrm{c}$ & $25.30 \mathrm{c}$ & $42.89 \mathrm{c}$ & $51.06 \mathrm{c}$ & $93.07 \mathrm{c}$ & $95.28 \mathrm{c}$ \\
\hline 110 & $99.21 \mathrm{~b}$ & $100.95 b$ & $22.03 b$ & $23.78 b$ & $3.96 \mathrm{c}$ & $4.42 b$ & $22.57 \mathrm{~b}$ & $23.11 \mathrm{~b}$ & $10.470 \mathrm{~b}$ & $11.060 \mathrm{~b}$ & $25.98 b$ & $26.70 \mathrm{~b}$ & $44.16 b$ & $51.95 b$ & $95.04 \mathrm{~b}$ & $97.05 b$ \\
\hline 165 & $105.33 a$ & $108.77 \mathrm{a}$ & $25.52 \mathrm{a}$ & $26.83 a$ & $4.43 \mathrm{a}$ & $4.80 \mathrm{a}$ & $23.96 a$ & $24.50 \mathrm{a}$ & $11.040 \mathrm{a}$ & $11.780 \mathrm{a}$ & $26.90 \mathrm{a}$ & $27.94 a$ & $46.18 \mathrm{a}$ & $53.26 a$ & $96.03 a$ & $98.02 \mathrm{a}$ \\
\hline F-test & $* *$ & $* *$ & $* *$ & $* *$ & $* *$ & $* *$ & $* *$ & $* *$ & $* *$ & $* *$ & $* *$ & $* *$ & $* *$ & $* *$ & $* *$ & $* *$ \\
\hline \multicolumn{17}{|l|}{ Interaction } \\
\hline $\mathrm{H} \times \mathrm{S}$ & $* *$ & $* *$ & $* *$ & $* *$ & $* *$ & $* *$ & $* *$ & $* *$ & $* *$ & $* *$ & $* *$ & $* *$ & $* *$ & $* *$ & $* *$ & $* *$ \\
\hline $\mathrm{H} \times \mathrm{N}$ & $* *$ & $* *$ & $* *$ & $* *$ & $* *$ & $* *$ & $* *$ & $* *$ & $* *$ & $* *$ & $* *$ & $* *$ & $* *$ & $* *$ & $* *$ & $* *$ \\
\hline $\mathrm{S} \times \mathrm{N}$ & $* *$ & $* *$ & $* *$ & $* *$ & $* *$ & $* *$ & $* *$ & $* *$ & $* *$ & $* *$ & $* *$ & $* *$ & $* *$ & $* *$ & $* *$ & $* *$ \\
\hline $\mathrm{H} \times \mathrm{S} \times \mathrm{N}$ & $* *$ & ** & $* *$ & $* *$ & $* *$ & $* *$ & ** & $* *$ & ** & ** & ** & $* *$ & $* *$ & $* *$ & $* *$ & $* *$ \\
\hline
\end{tabular}

** : highly significant at the $1 \%$ level of probability .In each season, the values having the same letter(s) not significantly differed according to Duncan's multiple range test. 
Table 2. Plant height $(\mathrm{cm})$, panicles hill ${ }^{-1}$, panicle weight $(\mathrm{g})$, panicle length $(\mathrm{cm})$, grain yield ( tha ${ }^{-1)}$, 1000 -grain weight $(\mathrm{g})$, harvest index $(\%)$ and spikelet fertility $(\%)$ as affected by the interaction between, hybrid and seedling ages during 2014 and 2015 seasons.

\begin{tabular}{|c|c|c|c|c|c|c|c|c|c|c|c|c|c|c|c|c|c|}
\hline \multirow[t]{2}{*}{ Hybrids } & \multirow{2}{*}{$\begin{array}{c}\text { Seedling } \\
\text { ages (day) }\end{array}$} & \multicolumn{2}{|c|}{ Plant height $(\mathrm{cm})$} & \multicolumn{2}{|c|}{ Panicles hill $^{-1}$} & \multicolumn{2}{|c|}{$\begin{array}{l}\text { Panicle weight } \\
(\mathrm{g})\end{array}$} & \multicolumn{2}{|c|}{$\begin{array}{l}\text { Panicle length } \\
(\mathrm{cm})\end{array}$} & \multicolumn{2}{|c|}{$\begin{array}{c}\text { Grain yield } \\
\left(\text { tha }^{-1}\right)\end{array}$} & \multicolumn{2}{|c|}{$\begin{array}{l}\text { 1000-grain weight } \\
(\mathrm{g})\end{array}$} & \multicolumn{2}{|c|}{$\begin{array}{c}\text { Harvest Index } \\
(\%)\end{array}$} & \multicolumn{2}{|c|}{$\begin{array}{c}\text { Spikelet fertility } \\
(\%)\end{array}$} \\
\hline & & 2014 & 2015 & 2014 & 2015 & 2014 & 2015 & 2014 & 2015 & 2014 & 2015 & 2014 & 2015 & 2014 & 2015 & 2014 & 2015 \\
\hline \multirow{3}{*}{$\begin{array}{l}\text { Egyptian } \\
\text { Hybrid } 1\end{array}$} & 20 & 101.5 & 104.10 & 21.48 & 23.11 & 4.30 & 4.70 & 22.36 & 23.53 & 10.670 & 11.343 & 25.60 & 24.23 & 43.64 & 51.22 & 94.32 & 96.28 \\
\hline & 25 & 100.40 & 102.39 & 19.72 & 21.06 & 4.04 & 4.24 & 21.81 & 22.74 & 9.587 & 10.392 & 25.25 & 23.92 & 41.81 & 48.07 & 94.13 & 96.17 \\
\hline & 30 & 98.19 & 100.41 & 18.33 & 19.47 & 3.66 & 3.95 & 21.30 & 21.77 & 8.372 & 9.347 & 24.84 & 22.95 & 39.99 & 45.1 & 92.49 & 94.52 \\
\hline \multirow{3}{*}{ SK2151H } & 20 & 95.40 & 97.08 & 20.50 & 21.97 & 3.92 & 4.27 & 21.85 & 22.60 & 9.383 & 9.970 & 26.59 & 26.75 & 42.41 & 49.17 & 95.70 & 97.73 \\
\hline & 25 & 93.55 & 96.52 & 18.97 & 20.23 & 3.57 & 3.86 & 21.32 & 21.75 & 8.571 & 9.224 & 26.11 & 26.1 & 40.85 & 45.77 & 94.44 & 96.43 \\
\hline & 30 & 93.29 & 94.87 & 16.57 & 18.04 & 3.22 & 3.48 & 20.47 & 20.89 & 7.803 & 8.469 & 25.03 & 25.4 & 39.32 & 43.93 & 93.06 & 95.4 \\
\hline \multicolumn{2}{|c|}{ LSD 0.05} & 0.6 & 0.7 & 0.02 & 0.02 & 0.02 & 6.6 & 0.01 & 0.01 & 0.02 & 0.02 & 0.02 & 2.2 & 0.01 & 6.57 & 0.1 & 5.8 \\
\hline \multicolumn{2}{|c|}{ LSD 0.01} & 0.8 & 0.1 & 0.03 & 0.03 & 0.03 & 9.5 & 0.02 & 0.02 & 0.03 & 0.03 & 0.03 & 3.2 & 0.02 & 9.51 & 0.2 & 8.4 \\
\hline
\end{tabular}

* and **significant differences at 0.05 and 0.01 level of probability respectively. 
Sarkar et al, (2011) found significant effect of seedling age of panicle length, they recorded more panicle length $(27.98 \mathrm{~cm})$ from seedlings of 25 days age. While minimum $(27.36 \mathrm{~cm})$ from older seedlings of 35 days. (Prabha et al, 2011) reported that, more panicle length $(24.1 \mathrm{~cm})$ by transplanting younger seedlings of 14 days age compared with older seedlings of 21 days which received minimum panicle length of $18.8 \mathrm{~cm}$ in system of rice intensification, IRRI (2014), reported that, transplanting at 21- day-old seedling ensures timely heading and flowering of parental lines. Transplanting older seedling delays flowering by about half the number of days by which the seedling are older than 21days.it transplanting younger seedling advanced flowering by approximately half the number of days by which the seedling are younger than 21 days. It transplanting the $\mathrm{B}$ or $\mathrm{R}$ line seedling by the same number of days synchronize flowering.

The results listed in Table (3) indicates that, the Egyptian Hybrid 1 and $165 \mathrm{~kg} \mathrm{~N} / \mathrm{ha}$ interaction revealed that significantly highest values for plant height $(\mathrm{cm})$, panicles hill ${ }^{-1}$, panicle weight $(\mathrm{gm})$, panicle length $(\mathrm{cm})$, grain yield $\left(\right.$ tha $\left.^{-1}\right), 1000$-grain weight $(\mathrm{g})$, harvest index $(\%)$ and spikelet fertility $(\%)$ in both seasons, where the values were 110.37 $(\mathrm{cm}), 28.02(\mathrm{~cm}), 5.17(\mathrm{gm}), 25.06(\mathrm{~cm}), 12.323$ (tha $^{-}$ $\left.{ }^{1}\right), 51.16(\%)$ and $97.90(\%)$ in the second seasons , respectively. While, the significantly lowest values were detected as a result of the hybrid SK $2151 \mathrm{H}$ without nitrogen application interaction for these traits in both seasons. Also, the highest value of 1000 -grain weight was $28.17(\mathrm{~g})$ when using $165 \mathrm{~kg}$ $\mathrm{N}$ /ha with the hybrid cross SK $2151 \mathrm{H}$ during season 2015 , but, the lowest value was 23.90 (g) obtained by without application nitrogen when interacted with Egyptian Hybrid 1 during 2014 season.

The results in Table 4. Indicates that, the seedling age 20 days $\times 165 \mathrm{~kg} \mathrm{~N} /$ ha interaction revealed the significantly highest values for the all traits in both seasons. Where the values were: $110.06(\mathrm{~cm}), 29.0$ hill $^{-1}, 5.50(\mathrm{~g}), 26.35(\mathrm{~cm}), 12.700$ tha $^{-1}, 28.7(\mathrm{~cm})$, $53.75(\%)$ and $99.1(\%)$ in the second season, respectively. While, the significantly lowest values were detected as a result of the 30 days seedling age and $0 \mathrm{~kg} \mathrm{~N} /$ ha interaction for the all traits in both seasons. Pramanik and Bera, 2013, evaluated hybrid rice to three levels of seedling age and five levels of nitrogen. They observed that 1000-grain weight affected significantly by different seedling age and nitrogen rates. Young seedlings of 10 days age produced maximum thousand grain weight $23.80 \mathrm{~g}$ while minimum $22.86 \mathrm{~g}$ was recorded from older seedlings of 30 days age. Thousand grain weight increased gradually with increase in nitrogen rate from 0 to $150 \mathrm{~kg} \cdot \mathrm{ha}^{-1}$ maximum 1000 -grain weight $24.11(\mathrm{~g})$ was obtained with the application of $150 \mathrm{~kg}$ $\mathrm{N} . \mathrm{ha}^{-1}$ and it decreased (to $23.87 \mathrm{~g}$ ) with further increase in nitrogen up to $200 \mathrm{~kg} \cdot \mathrm{ha}^{-1}$.

The results in Table 5. Indicates that, second order interaction of the hybrid combination Egyptian Hybrid 1 x 20 days seedling age and the $\mathrm{N}$ rate of $165 \mathrm{~kg} /$ ha revealed significantly highest values of plant height $(\mathrm{cm})$, panicles hill ${ }^{-1}$, panicle weight $(\mathrm{gm})$, panicle length $(\mathrm{cm})$, grain yield $\left(\right.$ tha $\left.^{-1}\right)$, harvest index (\%) and spikelet fertility (\%) during the two seasons. The highest values were $112.31(\mathrm{~cm}), 30.13$ hill ${ }^{-1}, 5.90(\mathrm{~g}), 26.30(\mathrm{~cm}), 13.600\left(\mathrm{tha}^{-1}\right), 51.2(\%)$ and $99.01(\%)$ for the seven traits in the second season, respectively. While the lowest values for these traits obtained when detected as a result of 30 days seedling age, hybrid SK2151 $\mathrm{H}$ and $0 \mathrm{~kg} \mathrm{~N} / \mathrm{ha}$. The highest value of 1000 -grain weight $(\mathrm{g})$ was 29.40 (g) was detected with hybrid SK2151H x 20 days seedling age and the $\mathrm{N}$ rate of $165 \mathrm{~kg} / \mathrm{ha}$. During season 2015, the lowest value was 23.9 (g) obtained from using the Egyptian Hybrid 1, seedling age 30 days and $0 \mathrm{~kg} \mathrm{~N} / \mathrm{ha}$ during 2014 season. Kaushal et al, 2010, recorded taller plants $115.6 \mathrm{~cm}$ when nitrogen was applied at $150 \mathrm{~kg} \mathrm{ha}^{-1}$ while minimum $104.1 \mathrm{~cm}$ was obtained in case of 90 $\mathrm{kg} \cdot \mathrm{N} \cdot \mathrm{ha}^{-1}$. Ehsanullah et al, 2012, reported that plant height had increased gradually with the increase in nitrogen fertilization to Basmati rice. Maximum plant height $107.60 \mathrm{~cm}$ was recorded when nitrogen was applied at $125 \mathrm{~kg} \cdot \mathrm{ha}-1$, while minimum plant height was $100.6 \mathrm{~cm}$ obtained when $75 \mathrm{~kg} \mathrm{~N} \cdot \mathrm{ha}^{-1}$ was applied. Pramanik and Bera, 2013, found significant effect of seedling age and nitrogen fertilization on plant height of hybrid rice. They found maximum plant height $(103.81 \mathrm{~cm}$ and $112.84 \mathrm{~cm})$ when 10 days old seedlings were transplanted and nitrogen was applied at $200 \mathrm{~kg} \cdot \mathrm{ha}^{-1}$, while minimum plant height $(91.38 \mathrm{~cm}$ and $81.63 \mathrm{~cm})$ was observed with the seedlings of 30 days age and with no nitrogen application. 
Table 3. Plant height $(\mathrm{cm})$, panicles hill ${ }^{-1}$, panicle weight $(\mathrm{g})$, panicle length $(\mathrm{cm})$, grain yield $\left(\right.$ tha $\left.^{-1}\right), 1000$-grain weight $(\mathrm{g})$, harvest index $(\%)$ and spikelet fertility $(\%)$ as affected by the interaction between seedling age and $\mathrm{N}$ rates during 2014 and 2015 seasons.

\begin{tabular}{|c|c|c|c|c|c|c|c|c|c|c|c|c|c|c|c|c|c|}
\hline \multirow{2}{*}{ Hybrids } & \multirow{2}{*}{$\begin{array}{l}\text { N-rates } \\
(\mathrm{kgN} / \mathrm{ha})\end{array}$} & \multicolumn{2}{|c|}{ Plant height $(\mathrm{cm})$} & \multicolumn{2}{|c|}{ Panicles hill-1 $^{-1}$} & \multicolumn{2}{|c|}{ Panicle weight (g) } & \multicolumn{2}{|c|}{$\begin{array}{l}\text { Panicle length } \\
(\mathrm{cm})\end{array}$} & \multicolumn{2}{|c|}{ Grain yield tha ${ }^{-1}$} & \multicolumn{2}{|c|}{$\begin{array}{c}\text { 1000-grain } \\
\text { weight }(\mathrm{g})\end{array}$} & \multicolumn{2}{|c|}{$\begin{array}{c}\text { Harvest Index } \\
(\%)\end{array}$} & \multicolumn{2}{|c|}{$\begin{array}{c}\text { Spikelet } \\
\text { fertility }(\%)\end{array}$} \\
\hline & & 2014 & 2015 & 2014 & 2015 & 2014 & 2015 & 2014 & 2015 & 2014 & 2015 & 2014 & 2015 & 2014 & 2015 & 2014 & 2015 \\
\hline \multirow{4}{*}{$\begin{array}{l}\text { Egyptian } \\
\text { Hybrid } 1\end{array}$} & 0 & 94.57 & 95.77 & 12.19 & 13.93 & 3.40 & 3.53 & 18.21 & 19.90 & 5.500 & 6.405 & 23.98 & 24.20 & 32.80 & 34.50 & 91.60 & 93.6 \\
\hline & 55 & 95.49 & 97.12 & 17.30 & 18.50 & 3.67 & 3.86 & 22.06 & 22.42 & 10.370 & 11.138 & 24.66 & 25.00 & 43.35 & 47.33 & 92.46 & 94.55 \\
\hline & 110 & 102.84 & 105.37 & 23.24 & 24.40 & 4.10 & 4.73 & 22.71 & 23.36 & 10.920 & 11.576 & 25.64 & 26.20 & 44.30 & 50.23 & 94.51 & 96.52 \\
\hline & 165 & 107.20 & 110.73 & 26.70 & 28.02 & 4.83 & 5.17 & 24.36 & 25.06 & 11.360 & 12.323 & 26.63 & 27.54 & 46.80 & 51.16 & 96.02 & 97.9 \\
\hline \multirow{2}{*}{ SK2151H } & 110 & 95.57 & 96.52 & 20.84 & 23.17 & 3.82 & 4.11 & 22.43 & 22.87 & 10.020 & 10.543 & 26.32 & 27.30 & 44.03 & 47.67 & 95.57 & 97.57 \\
\hline & 165 & 103.46 & 106.82 & 24.35 & 25.65 & 4.03 & 4.43 & 23.57 & 23.94 & 10.720 & 11.236 & 27.17 & 28.17 & 45.56 & 48.36 & 96.04 & 98.07 \\
\hline \multicolumn{2}{|c|}{ LSD 0.05} & 0.7 & 0.8 & 0.02 & 0.03 & 0.02 & 7.6 & 0.02 & 0.02 & 0.03 & 0.03 & 0.02 & 2.5 & 0.02 & 7.6 & 0.1 & 6.7 \\
\hline \multicolumn{2}{|c|}{ LSD 0.01} & 0.9 & 1.15 & 0.03 & 0.04 & 0.03 & 0.01 & 0.02 & 0.03 & 0.04 & 0.04 & 0.04 & 3.7 & 0.03 & 0.01 & 0.17 & 9.7 \\
\hline
\end{tabular}

$*$ and $* *$ significant differences at 0.05 and 0.01 level of probability respectively. 
Table 4. Plant height $(\mathrm{cm})$, panicles hill ${ }^{-1}$, panicle weight $(\mathrm{g})$, panicle length $(\mathrm{cm})$, grain yield tha ${ }^{-1}, 1000$-grain weight $(\mathrm{g})$, harvest index $(\%)$ and spikelet fertility $(\%)$ as affected by the interaction between seedling age and $\mathrm{N}$ rates during 2014 and 2015 seasons.

\begin{tabular}{|c|c|c|c|c|c|c|c|c|c|c|c|c|c|c|c|c|c|}
\hline \multirow{2}{*}{$\begin{array}{c}\text { Seedling } \\
\text { ages (day) }\end{array}$} & \multirow{2}{*}{$\begin{array}{l}\text { N-rates } \\
(\mathrm{kgN} / \mathrm{ha})\end{array}$} & \multicolumn{2}{|c|}{$\begin{array}{l}\text { Plant height } \\
(\mathrm{cm})\end{array}$} & \multicolumn{2}{|c|}{ Panicles hill ${ }^{-1}$} & \multicolumn{2}{|c|}{$\begin{array}{c}\text { Panicle } \\
\text { weight }(\mathrm{g})\end{array}$} & \multicolumn{2}{|c|}{$\begin{array}{l}\text { Panicle length } \\
(\mathrm{cm})\end{array}$} & \multicolumn{2}{|c|}{$\begin{array}{c}\text { Grain yield } \\
\left(\text { tha }^{-1}\right)\end{array}$} & \multicolumn{2}{|c|}{$\begin{array}{c}\text { 1000-grain } \\
\text { weight }(\mathrm{g})\end{array}$} & \multicolumn{2}{|c|}{$\begin{array}{c}\text { Harvest Index } \\
(\%)\end{array}$} & \multicolumn{2}{|c|}{$\begin{array}{c}\text { Spikelet fertility } \\
(\%)\end{array}$} \\
\hline & & 2014 & 2015 & 2014 & 2015 & 2014 & 2015 & 2014 & 2015 & 2014 & 2015 & 2014 & 2015 & 2014 & 2015 & 2014 & 2015 \\
\hline \multirow{4}{*}{20} & 0 & 90.30 & 91.41 & 12.65 & 14.50 & 3.50 & 3.70 & 18.55 & 20.40 & 6.138 & 6.898 & 24.51 & 24.79 & 33.63 & 35.55 & 92.86 & 94.85 \\
\hline & 55 & 95.26 & 97.38 & 20.02 & 21.10 & 3.75 & 3.90 & 22.20 & 22.80 & 10.565 & 11.239 & 25.52 & 25.95 & 43.98 & 47.36 & 94.02 & 96.03 \\
\hline & 110 & 101.10 & 103.50 & 23.65 & 25.51 & 4.35 & 4.85 & 23.07 & 23.72 & 11.391 & 11.735 & 26.50 & 27.40 & 46.10 & 49.15 & 96.03 & 98.04 \\
\hline & 165 & 107.13 & 110.06 & 27.63 & 29.00 & 4.85 & 5.50 & 24.60 & 26.35 & 12.013 & 12.700 & 27.85 & 28.7 & 48.40 & 53.75 & 97.13 & 99.1 \\
\hline \multirow{4}{*}{25} & 0 & 89.21 & 91.56 & 11.56 & 13.20 & 3.26 & 3.45 & 17.77 & 19.01 & 4.871 & 5.963 & 24.40 & 24.6 & 31.86 & 32.04 & 92.06 & 94.01 \\
\hline & 55 & 94.43 & 96.23 & 17.80 & 18.53 & 3.48 & 3.66 & 21.85 & 22.19 & 9.851 & 10.540 & 25.30 & 25.42 & 43.08 & 47.05 & 93.34 & 95.45 \\
\hline & 110 & 98.58 & 100.79 & $22 . .45$ & 23.90 & 3.98 & 4.40 & 22.75 & 23.25 & 10.505 & 11.125 & 26.18 & 26.75 & 44.30 & 48.0 & 95.30 & 97.3 \\
\hline & 165 & 105.68 & 109.23 & 25.65 & 26.96 & 4.50 & 4.70 & 23.90 & 24.55 & 11.090 & 11.605 & 26.85 & 27.80 & 46.10 & 50.02 & 96.45 & 98.45 \\
\hline \multirow{4}{*}{30} & 0 & 88.43 & 90.33 & 10.80 & 12.00 & 3.05 & 3.25 & 17.00 & 17.92 & 4.008 & 4.558 & 23.91 & 24.01 & 30.88 & 30.1 & 90.93 & 93.2 \\
\hline & 55 & 93.39 & 94.67 & 15.70 & 16.60 & 3.23 & 3.40 & 21.25 & 21.45 & 8.786 & 9.720 & 24.58 & 24.85 & 41.60 & 45.8 & 91.86 & 94.35 \\
\hline & 110 & 97.95 & 98.56 & 20.01 & 21.95 & 3.55 & 4.02 & 21.90 & 22.35 & 9.518 & 10.320 & 25.23 & 26.10 & 42.10 & 46.06 & 93.81 & 95.8 \\
\hline & 165 & 103.18 & 107.01 & 23.30 & 24.47 & 3.95 & 4.19 & 23.40 & 23.60 & 10.038 & 11.00 & 26.01 & 27.05 & 44.05 & 48.1 & 94.51 & 96.51 \\
\hline \multicolumn{2}{|c|}{ LSD 0.05} & 0.8 & 0.9 & 0.03 & 0.03 & 0.03 & 9.3 & 0.02 & 0.03 & 0.03 & 0.03 & 0.03 & 3.09 & 0.03 & 9.29 & 0.03 & 8.19 \\
\hline \multicolumn{2}{|c|}{ LSD 0.01} & 1.2 & 1.4 & 0.04 & 0.04 & 0.04 & 0.01 & 0.03 & 0.04 & 0.04 & 0.04 & 0.04 & 4.5 & 0.03 & 0.01 & 0.04 & 0.01 \\
\hline
\end{tabular}

\footnotetext{
$*$ and $* *$ significant differences at 0.05 and 0.01 level of probability respectively.
} 
Table 5. Plant height $(\mathrm{cm})$, panicles hill ${ }^{-1}$, panicle weight $(\mathrm{gm})$, panicle length $(\mathrm{cm})$, grain yield tha ${ }^{-1}, 1000$-grain weight $(\mathrm{g})$, harvest index $(\%)$ and spikelet fertility $(\%)$ as affected by the interaction among hybrid combination, seedling ages, and N-rates in 2014 and 2015 seasons.

\begin{tabular}{|c|c|c|c|c|c|c|c|c|c|c|c|c|c|c|c|c|c|c|}
\hline \multirow[t]{2}{*}{ Hybrids } & \multirow{2}{*}{$\begin{array}{c}\text { Seedlig } \\
\text { ages } \\
\text { (day) }\end{array}$} & \multirow{2}{*}{$\begin{array}{l}\text { N-rates } \\
(\mathrm{kgN} / \mathrm{ha})\end{array}$} & \multicolumn{2}{|c|}{ Plant height $(\mathrm{cm})$} & \multicolumn{2}{|c|}{ Panicles hill $^{-1}$} & \multicolumn{2}{|c|}{$\begin{array}{c}\text { Panicle weight } \\
(\mathrm{g})\end{array}$} & \multicolumn{2}{|c|}{$\begin{array}{l}\text { Panicle length } \\
(\mathrm{cm})\end{array}$} & \multicolumn{2}{|c|}{$\begin{array}{c}\text { Grain yield } \\
\left(\text { tha }^{-1}\right)\end{array}$} & \multicolumn{2}{|c|}{$\begin{array}{l}\text { 1000-grain } \\
\text { weight }(\mathrm{g})\end{array}$} & \multicolumn{2}{|c|}{$\begin{array}{c}\text { Harvest Index } \\
(\%)\end{array}$} & \multicolumn{2}{|c|}{$\begin{array}{c}\text { Spikelet fertility } \\
(\%)\end{array}$} \\
\hline & & & 2014 & 2015 & 2014 & 2015 & 2014 & 2015 & 2014 & 2015 & 2014 & 2015 & 2014 & 2015 & 2014 & 2015 & 2014 & 2015 \\
\hline \multirow{12}{*}{$\begin{array}{l}\text { Egyptian } \\
\text { Hybrid } 1\end{array}$} & \multirow{4}{*}{20} & 0 & 95.50 & 97.50 & 13.06 & 15.00 & 3.61 & 3.80 & 19.10 & 21. & 6.900 & 7.316 & 24.01 & 24.39 & 34.16 & 36.50 & 92.02 & 94.02 \\
\hline & & 55 & 96.40 & 98.76 & 19.77 & 21.30 & 3.90 & 4.01 & 22.30 & 23.10 & 11.280 & 12.036 & 25.00 & 25.50 & 44.80 & 47.80 & 93.40 & 95.05 \\
\hline & & 110 & 105.02 & 108.01 & 24.30 & 26.01 & 4.50 & 5.10 & 23.15 & 24.05 & 11.870 & 12.420 & 26.01 & 26.80 & 46.40 & 49.4 & 95.05 & 97.08 \\
\hline & & 165 & 109.03 & 112.31 & 28.80 & 30.13 & 5.20 & 5.90 & 24.90 & 26.03 & 12.620 & 13.600 & 27.40 & 28.01 & 49.20 & 51.2 & 97.17 & 99.01 \\
\hline & \multirow{4}{*}{25} & 0 & 94.43 & 95.63 & 12.01 & 13.80 & 3.40 & 3.50 & 18.05 & 20 & 5.510 & 6.800 & 24.00 & 24.20 & 32.6 & 32.6 & 92.10 & 94.01 \\
\hline & & 55 & 95.90 & 96.90 & 16.90 & 18.01 & 3.66 & 3.77 & 22.10 & 22.38 & 10.470 & 11.070 & 24.70 & 25.01 & 43.67 & 45.7 & 92.65 & 94.9 \\
\hline & & 110 & 103.06 & 106.0 & 23.40 & 24.30 & 4.10 & 4.70 & 22.80 & 23.50 & 10.960 & 11.500 & 25.80 & 26.10 & 44.40 & 48.4 & 94.90 & 96.9 \\
\hline & & 165 & 108.20 & 111.01 & 26.70 & 28.13 & 5.00 & 5.01 & 24.30 & 25.10 & 11.410 & 12.200 & 26.50 & 27.60 & 46.60 & 50.6 & 96.90 & 98.8 \\
\hline & \multirow{4}{*}{30} & 0 & 93.80 & 94.8 & 11.50 & 13.01 & 3.20 & 3.30 & 17.50 & 18.70 & 4.110 & 5.100 & 23.95 & 24.01 & 31.66 & 29.7 & 90.66 & 92.8 \\
\hline & & 55 & 94.19 & 95.7 & 15.23 & 16.200 & 3.46 & 3.50 & 21.60 & 21.79 & 9.370 & 10.310 & 24.30 & 24.50 & 41.60 & 44.6 & 91.70 & 93.7 \\
\hline & & 110 & 100.40 & 102.1 & 22.01 & 22.90 & 3.70 & 4.40 & 22.20 & 22.50 & 9.930 & 10.800 & 25.11 & 25.70 & 42.10 & 46.1 & 93.60 & 95.6 \\
\hline & & 165 & 104.36 & 109.0 & 24.60 & 25.80 & 4.30 & 4.60 & 23.90 & 24.10 & 10.60 & 11.170 & 26.01 & 27.01 & 44.50 & 49.6 & 94.01 & 96.01 \\
\hline \multirow{12}{*}{ SK215H } & \multirow{4}{*}{20} & 0 & 85.11 & 87.01 & 12.25 & 14.00 & 3.40 & 3.60 & 18.01 & 19.80 & 5.380 & 6.480 & 25.01 & 25.20 & 33.10 & 34.1 & 93.70 & 95.7 \\
\hline & & 55 & 94.13 & 96.01 & 20.28 & 20.90 & 3.60 & 3.80 & 22.10 & 22.50 & 9.850 & 10.550 & 26.04 & 26.40 & 43.17 & 46.7 & 95.01 & 97.02 \\
\hline & & 110 & 97.13 & 99.1 & 23.01 & 25.00 & 4.20 & 4.60 & 23 & 23.40 & 10.910 & 11.050 & 27.00 & 28.0 & 45.80 & 48.8 & 97 & 99.01 \\
\hline & & 165 & 105.23 & 108.01 & 26.46 & 28.0 & 4.50 & 5.10 & 24.30 & 24.70 & 11.420 & 11.800 & 28.30 & 29.40 & 47.60 & 50.6 & 97.10 & 99.2 \\
\hline & \multirow{4}{*}{25} & 0 & 84.00 & 85.8 & 11.11 & 12.60 & 3.12 & 3.40 & 17.60 & 18.01 & 4.230 & 5.126 & 24.80 & 25.0 & 31.12 & 32.1 & 92.3 & 94.02 \\
\hline & & 55 & 92.96 & 95.5 & 18.70 & 19.05 & 3.30 & 3.55 & 21.60 & 21.99 & 9.230 & 10.010 & 25.90 & 26.05 & 42.50 & 45.5 & 94.20 & 96.01 \\
\hline & & 110 & 94.10 & 95.2 & 21.50 & 23.30 & 3.86 & 4.10 & 22.70 & 23.11 & 10.050 & 10.750 & 26.56 & 27.40 & 44.20 & 47.2 & 95.7 & 97.7 \\
\hline & & 165 & 103.16 & 107.46 & 24.60 & 25.40 & 4.01 & 4.40 & 23.50 & 24.00 & 10.770 & 11.00 & 27.20 & 28.01 & 45.60 & 48.6 & 96 & 98.01 \\
\hline & \multirow{4}{*}{30} & 0 & 83.06 & 85.86 & 10.10 & 11.0 & 2.90 & 3.20 & 16.50 & 17.14 & 3.900 & 4.020 & 23.86 & 24.0 & 30.10 & 38.1 & 91.20 & 93.6 \\
\hline & & 55 & 92.60 & 93.63 & 16.18 & 17.0 & 3.01 & 3.30 & 20.90 & 21.11 & 8.200 & 9.136 & 24.80 & 25.20 & 41.60 & 44.6 & 92.3 & 95.01 \\
\hline & & 110 & 95.50 & 95.0 & 18.01 & 21.01 & 3.40 & 3.64 & 21.68 & 22.20 & 9.100 & 9.836 & 25.40 & 26.50 & 42.10 & 46.1 & 94.2 & 96.02 \\
\hline & & 165 & 102.00 & 105.0 & 22.01 & 22.15 & 3.60 & 3.79 & 22.91 & 23.11 & 10.010 & 10.300 & 26.01 & 27.10 & 43.50 & 47.5 & 95 & 97.02 \\
\hline \multicolumn{2}{|c|}{ LSD 0.05} & & 1.2 & 1.4 & 0.04 & 0.04 & 0.04 & 0.01 & 0.03 & 0.04 & 0.04 & 0.03 & 0.03 & 4.4 & 0.04 & 0.01 & 0.02 & 0.01 \\
\hline \multicolumn{2}{|c|}{ LSD 0.01} & & 1.7 & 1.1 & 0.06 & 0.06 & 0.06 & 0.02 & 0.04 & 0.05 & 0.06 & 0.06 & 0.06 & 6.3 & 0.05 & 0.02 & 0.31 & 0.02 \\
\hline
\end{tabular}




\section{References}

Bastawisi, A.O.; Aidy, I .R.; El-Mowafy, H.F. and Maximos, M.A. (1998). Research and development for hybrid rice technology in Egypt. In Advances of hybrid rice technology. Proceedings of the $3^{\text {rd }}$ International Symposium, 14-16 Nov. Hyderabad, India: International Rice Research Institute. Manila (Philippines) 367-372.

Ehsanullah, K. Jabran ; Asghar, G.; Hussain, M. and Rafiq, M. (2012). Effect of nitrogen fertilization and seedling density on fine rice yield in Faisalabad, Pakistan. Soil \& Environment, 31, 152-156.

Faghani, R.; Mobasser, H.R.; Dehpor, A.A. and Kochaksarai, S.T. (2011). The effect of planting date and seedling age on yield and yield components of rice (Oryza sativa L.) varieties in north of Iran. African Journal of Agricultural Research, 6, 2571-2575.

Gomez, K.A. and Gomez, A.A. (1984). Statistical Procedures for Agricultural Research. 2 ${ }^{\text {nd }}$ Ed. John Wiley and Sons, Inc. New York.

Hasanuzzaman, M.; Ali, M.H.; Karim, M. F.; Masum, S.M. and Mahmud, J.A. (2012). Response of hybrid rice to different levels of nitrogen and phosphorus. International Research Journal of Applied and Basic Sciences, 3, 25222528.

Hosseiny, Y. and Maftoun, M. (2008). Effects of nitrogen levels, nitrogen sources and zinc rates on the growth and mineral composition of lowland rice. J. Agric. Sci. Technol. (2008) Vol. 10: 307316.

IRRI (2014) International Rice research descriptors for rice. Los B. anos, Laguna, Philippines.52p.

Kandil, A.A.; El-Kalla, S.E.; Badawi, A.T. and ElShayb, O.M. (2010). Effect of hill spacing, nitrogen levels and harvest date on rice productivity and grain quality. Crop \& Environment, 1, 22-26.
Kaushal, A.K.; N.S. Rana.; A.Singh.; S.Neeray and A.Strivastav (2010). Response of levels and split application of nitrogen in green manured wetland rice (Oryza sativa L.). Asian Journal of Agricultural Sciences, 2, 42-46.

Metwally, T.F.; E.E. Gewaily and S.S. Naeem (2011). Nitrogen response curve and nitrogen use efficiency of egyptian hybrid rice. Journal of Agricultural Research, 37, 73-84.

Molla , M.A.H.(2002). Influence of seedling age and number of seedlings on yield attributes and yield of hybrid rice in the wet season .International Rice Research Notes . 26:2, 73-74.

Prabha, A.C.; Thiyagrajan, T.M. and M. Senthivelu (2011). System of rice intensification principles on growth parameters, yield attributes and yield of rice (Oryza sativa L.). Journal of Agronomy, 10, 27-33.

Pramanik, K. and Bera, A. K. (2013). Effect of seedling age and nitrogen fertilizer on growth, chlorophyll content, yield and economics of hybrid rice (Oryza sativa L). International Journal of Agronomy and Plant Production, 4, 34893499.

Sarkar, M.A.R.; Paul, S.K. and Hossain, M.A. (2011). Effect of row arrangement, age of tiller seedling and number of tiller seedlings per hill on performance of transplant aman rice. Journal of Agricultural Science, 6, 61-63.

Waghmode, B.D.; Ingale, B.V.; Dalvi,V.V. and Rewale, A.P. (2008). Effect of seedling age on 50per cent flowering of parental lines of sahyadri rice hybrid .Journal of Maharashtra Agriculture Universities .2008.33:1,28-30.4ref.

Yoseftabar, S. (2013). Effect nitrogen management on panicle structure and yield in rice (Oryza sativa L.). International Journal of Agriculture and Crop Sciences, 5, 1224-1227. 


\section{تأثير التسميد النتروجينى وعمر البادرة على انتاجية بعض هجن الآرز المبشرة حسن شحاته حمد \\ قسم بحوث الأرز - معهد بحوث المحاصيل الحقلية - مركز البحوث الزراعية - الجيزه - مصر.}

أجريت هذه الدراسة في مزرعة مركز البحوث الزراعية - محطة بحوث سخا - كفر الثيخ - مصر. خلال موسمي الزراعة

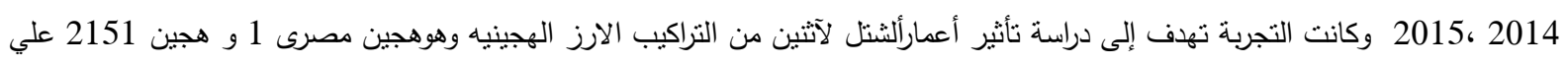

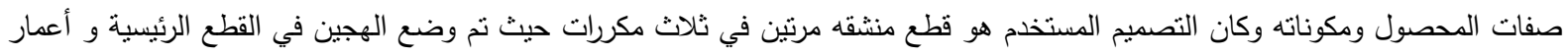

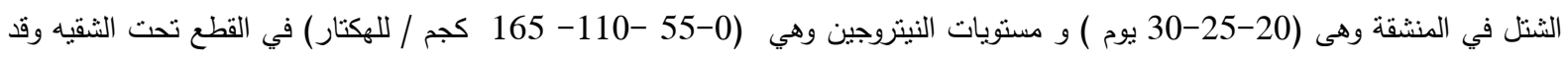

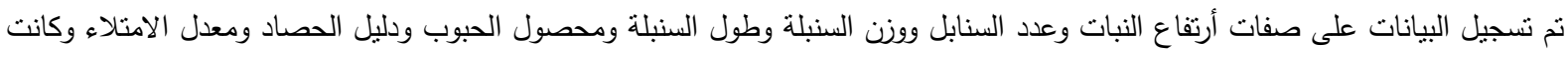

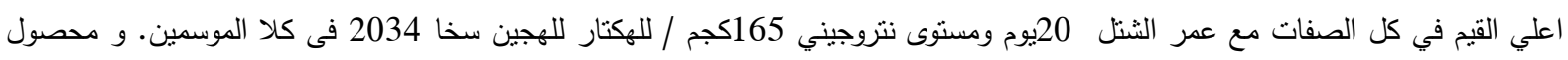
الحبوب كان600 و13طن/لاككتار بأستخدام 165كجم نتروجين / للهكتار مع عمر الثنل 20يوم للهجين مصرى1 فئ كلا الموسمين. 